\title{
WEIGHTED NORM INEQUALITIES FOR POTENTIALS WITH APPLICATIONS TO SCHRÖDINGER OPERATORS, FOURIER TRANSFORMS, AND CARLESON MEASURES
}

\author{
BY R. KERMAN ${ }^{1}$ AND E. SAWYER ${ }^{2}$
}

I. Introduction. A new characterization of the trace inequality for potential operators is given and used to sharpen recent results of C. L. Fefferman and D. H. Phong on the distribution of eigenvalues of Schrödinger operators. It is also used to study the domain and essential spectrum of Schrödinger operators, to obtain weighted norm inequalities for Fourier transforms, and to determine the Carleson measures for Dirichlet-type spaces.

THEOREM 1. Suppose $K$ is a nonnegative, locally integrable, radial function on $R^{n}$, which is decreasing as a function of $|x|$. For $f$ in the class $P\left(R^{n}\right)$ of nonnegative, measurable functions on $R^{n}$ and $x \in R^{n}$, set

$$
(T f)(x)=(K * f)(x)=\int_{R^{n}} K(x-y) f(y) d y,
$$

provided this integral exists for almost all $x \in R^{n}$. Then given $1<p<\infty$ and $v \in P\left(R^{n}\right)$, there exists $C>0$ so that the trace inequality

$$
\int_{R^{n}}(T f)(x)^{p} v(x) d x \leq C \int_{R^{n}} f(x)^{p} d x, \quad f \in P\left(R^{n}\right),
$$

holds if and only if $C^{\prime}>0$ exists with

$$
\int_{Q} T\left(\chi_{Q} v\right)(x)^{p^{\prime}} d x \leq C^{\prime} \int_{Q} v(x) d x<\infty \text { for all dyadic cubes } Q
$$

where, as usual, $p^{\prime}=p /(p-1)$.

Alternative characterizations of the trace inequality in terms of $L^{p}$ capacities have been obtained in [ $\mathbf{1}$ and $\mathbf{4}]$.

The trace inequality (1), for $p=2$, and the potential kernel $K^{\alpha}(x)$, with $\hat{K}^{\alpha}(\zeta)=\left(\alpha+|\zeta|^{2}\right)^{-1 / 2}$, arises in estimating the eigenvalues ${ }^{3}$ of a Schrödinger operator $H$. Let

$$
\left(I_{2} f\right)(x)=\int_{R^{n}}|x-y|^{2-n} f(y) d y
$$

Received by the editors March 26, 1984 and, in revised form, August 15, 1984.

1980 Mathematics Subject Classification. Primary 26D10, 42B25.

Key words and phrases. Weighted norm inequality, potential operator, Schrödinger operator, Fourier transform, Carleson measure.

${ }^{1}$ Research supported in part by N.S.E.R.C. grant A4021.

${ }^{2}$ Research supported in part by N.S.E.R.C. grant A5149.

${ }^{3}$ By eigenvalues we mean the numbers $\lambda_{1}<\cdots<\lambda_{N}<\cdots$, where $\lambda_{N}$ is the maximum over all $N-1$ tuples $\phi_{1}, \ldots, \phi_{N-1}$ of the quantity $\inf \langle H u, u\rangle /\langle u, u\rangle$, the infimum being over all $u \in Q(H), u \perp \phi_{j}, j=1, \ldots, N-1$. Here $Q(H)$ denotes the form domain of $H$. See $[10]$. 
denote the Newtonian potential of $f$. The following result refines the estimates of the least eigenvalue of $H$ given in Theorem 5 of $[3]$.

THEOREM 2. Let $H=-\Delta-v$, where $v \in P\left(R^{n}\right), n \geq 3$. Denote the $v$ measure of $Q, \int_{Q} v(x) d x$, by $|Q|_{v}$. There are positive constants $C$ and $c$, depending only on the dimension $n$, such that the least eigenvalue, $\lambda_{1}$, of $H$ satisfies $E_{\mathrm{sm}} \leq-\lambda_{1} \leq E_{\mathrm{big}}$, where

$$
\begin{aligned}
& E_{\mathrm{sm}}=\sup \left\{|Q|^{-2 / n}:|Q|_{v}^{-1} \int_{Q} I_{2}\left(\chi_{Q} v\right) v \geq C\right\} \\
& E_{\mathrm{big}}=\sup \left\{|Q|^{-2 / n}:|Q|_{v}^{-1} \int_{Q} I_{2}\left(\chi_{Q} v\right) v \geq c\right\}
\end{aligned}
$$

A similar refinement of Theorems 6 and $6^{\prime}$ in $[\mathbf{3}]$ is given in

THEOREM 3. Let $H=-\Delta-v$, where $v \in P\left(R^{n}\right), n \geq 3$. There are positive constants $C$ and $c$, depending only on the dimension $n$, such that

(A) $H$ has at least $N$ eigenvalues $\leq-\lambda, \lambda>0$, provided there exists a collection of $N$ cubes $Q_{1}, \ldots, Q_{N}$ of side length at most $\lambda^{-1 / 2}$, whose doubles are pairwise disjoint, with $\left|Q_{j}\right|_{v}^{-1} \int_{Q_{j}} I_{2}\left(\chi_{Q_{j}} v\right) v \geq C, 1 \leq j \leq N$.

Conversely,

(B) $H$ having at least $C N$ eigenvalues $\leq-\lambda$ implies there is a collection of $N$ pairwise disjoint dyadic cubes $Q_{1}, \ldots, Q_{N}$, of side length at most $\lambda^{-1 / 2}$, that satisfy

$$
\left|Q_{\jmath}\right|_{v}^{-1} \int_{Q_{\jmath}} I_{2}\left(\chi_{Q_{\jmath}} v\right) v \geq c, \quad 1 \leq j \leq N .
$$

REMARKS. 1. Roughly speaking, Theorem 3 says that the negative eigenvalues of $H$ are approximately given by $-|Q|^{-2 / n}$ as $Q$ ranges over all the minimal dyadic cubes satisfying $|Q|_{v}^{-1} \int_{Q} I_{2}\left(\chi_{Q} v\right) v \geq C$.

2. As an illustration of Theorem 2, consider Example $\mathrm{V}$ in [3]: a particle in a rectangular box $B=B_{1} \times B_{2} \times \cdots \times B_{n}$ with side lengths $\delta_{1} \leq \delta_{2} \leq \cdots \leq \delta_{n}$. Let $v=\chi_{B}$ and $x_{B}$ denote the centre of $B$. Since

$$
\begin{aligned}
\sup _{Q}|Q|_{v}^{-1} \int_{Q} I_{2}\left(\chi_{Q} v\right) v & \cong I_{2} v\left(x_{B}\right) \cong \delta_{1}^{2}+\delta_{1} \delta_{2}\left(1+\log \delta_{3} / \delta_{2}\right) \\
& \cong \delta_{1} \delta_{2} \log \left(1+\delta_{3} / \delta_{2}\right)
\end{aligned}
$$

Theorem 2 yields the correct order of magnitude for the energy, $E_{\text {critical }}$, needed to trap a particle in $B$, namely

$$
E_{\text {critical }}=\sup \{E \geq 0:-\Delta-E v \geq 0\} \cong\left(\delta_{1} \delta_{2} \log \left(1+\delta_{3} / \delta_{2}\right)\right)^{-1} .
$$

3. The quantity $|Q|_{v}^{-1} \int_{Q} I_{2}\left(\chi_{Q} v\right) v$ is, in a sense, intermediate between the simpler ones used in $[\mathbf{3}]$ for the results corresponding to (A) and (B). Indeed, it is possible to show that for $p>1$, 


$$
|Q|^{2 / n-1} \int_{Q} v \leq C|Q|_{v}^{-1} \int_{Q} I_{2}\left(\chi_{Q} v\right) v \leq C_{p} \sup _{Q^{\prime} \subset Q}\left|Q^{\prime}\right|^{2 / n-1 / p}\left(\int_{Q^{\prime}} v^{p}\right)^{1 / p}
$$

The trace inequality also arises in questions concerning the domain and essential spectrum of Schrödinger operators. For example, conditions like (2) determine when the operator $T$ in (1) is compact. This leads to conditions sufficient for $H$ to have the same essential spectrum as $-\Delta$, that is, $[0, \infty){ }^{4}$

Another application of Theorem 1 is to weighted inequalities for Fourier transforms on $R$.

THEOREM 4. Suppose $u(x)$ is an even, locally integrable function on $R$ which is convex and decreases to 0 on $(0, \infty)$. Then for arbitrary $v(x) \geq 0$,

$$
\int_{-\infty}^{\infty}|\hat{f}(x) u(x)|^{2} d x \leq C \int_{-\infty}^{\infty}|f(x) v(x)|^{2} d x \quad \text { for all } f \in L^{1}(R)
$$

if and only if

$$
\int_{I} \tilde{M}\left(\chi_{I} v^{-2}\right)(x)^{2} d x \leq C^{\prime} \int_{I} v(x)^{-2} d x \quad \text { for all intervals } I
$$

where

$$
(\tilde{M} f)(x)=\sup _{x \in I}\left[\int_{0}^{|I|^{-1}} u(y) d y\right] \int_{I}|f(y)| d y
$$

For earlier conditions guaranteeing (3) see [5, 6, and 7].

Our final application is to Carleson measures for the Dirichlet-type spaces $h_{K}^{p}$ introduced in [9]. The space $h_{K}^{p}$ consists of the Poisson integrals, $u$, of potentials $K * f, f \in L^{p}\left(R^{n}\right)$. A positive measure $\mu$ on $R_{+}^{n+1}$ is said to be a Carleson measure for $h_{K}^{p}$ if $\|u\|_{L^{p}(\mu)} \leq C\|f\|_{p}$ for all $f \in L^{p}\left(R^{n}\right)$.

THEOREM 5. Suppose $K(x)$ is nonnegative and radial on $R^{n}$ and is decreasing as a function of $|x|$. Then for $1<p<\infty$, a positive Borel measure $\mu$ on $R_{+}^{n+1}$ is a Carleson measure for $h_{K}^{p}$ if and only if

$$
\int_{Q} \bar{M}\left(\chi_{T(Q)} \mu\right)(x)^{p^{\prime}} d x \leq C \int_{T(Q)} d \mu<\infty \quad \text { for all cubes } Q
$$

Here, $Q$ is a cube in $R^{n}$ and $T(Q)$ denotes the cube in $R_{+}^{n+1}$ having $Q$ as a face. The Carleson maximal function, $\bar{M} \nu$, is given at $x \in R^{n}$ by

$$
\bar{M} \nu(x)=\sup _{x \in Q}\left[|Q|^{-1} \int_{|y| \leq|Q|^{1 / n}} K(y) d y\right] \int_{T(Q)} d \nu
$$

A characterization of Carleson measures in terms of $L^{p}$ capacities can be found in [9 and 12].

\footnotetext{
${ }^{4}$ We wish to thank M. Wilson for communicating to us an alternative proof of the connection between Theorem 1 and the results of $[\mathbf{3}]$.
} 


\section{Sketch of proofs.}

PROOF OF THEOREM 1 By duality, (1) is equivalent to

$$
\int T(g v)^{p^{\prime}} \leq C^{\prime} \int g^{p^{\prime}} v, \quad g \in P\left(R^{n}\right) .
$$

Extensions of theorems in [8] show that (4) amounts to the same inequality with $T$ replaced by the dyadic maximal operator

$$
(M f)(x)=\sup _{x \in Q}\left[|Q|^{-1} \int_{|y| \leq|Q|^{1 / n}} K(y) d y\right] \int_{Q}|f|, \quad Q \text { dyadic. }
$$

The methods of $[\mathbf{1 1}]$ now yield (2), with $M$ instead of $T$, as necessary and sufficient for the latter inequality. Finally, as above, $M$ and $T$ are interchangeable, so the proof is complete.

PROOF OF THEOREM 2. We have

$$
-\lambda_{1} \equiv \sup _{u \in Q(H)}-\frac{\langle H u, u\rangle}{\langle u, u\rangle}=\inf \left\{\alpha>0: C_{\alpha} \leq 1\right\},
$$

where $C_{\alpha}$ is the least constant such that $\int_{R^{n}}\left(I_{1}^{\alpha} f\right)^{2} v \leq C_{\alpha} \int f^{2}$ for all $f \in$ $P\left(R^{n}\right)$, and $I_{k}^{\alpha}$ has kernel $K_{k}^{\alpha}$ with $\hat{K}_{k}^{\alpha}(\zeta)=\left(\alpha+|\zeta|^{2}\right)^{-k / 2}$. This is so since

$$
\begin{aligned}
\sup _{u \in Q(H)} & -\frac{\langle H u, u\rangle}{\langle u, u\rangle}=\sup _{u \in Q(H)} \frac{\int|u|^{2} v-\int|\nabla u|^{2}}{\int|u|^{2}} \\
= & \inf \left\{\alpha>0: \int|u|^{2} v \leq \int\left(\alpha|u|^{2}+|\nabla u|^{2}\right)=\int\left(\alpha+|\zeta|^{2}\right)|\hat{u}(\zeta)|^{2} d \zeta\right\} \\
= & \inf \left\{\alpha>0: C_{\alpha} \leq 1\right\} .
\end{aligned}
$$

Theorem 1 now yields $C_{\alpha} \cong \sup _{Q}|Q|_{v}^{-1} \int I_{1}^{\alpha}\left(\chi_{Q} v\right)^{2}$. Standard estimates on Bessel kernels show it suffices to take this supremum over cubes of side length at most $\alpha^{-1 / 2}$, so Theorem 2 follows readily, since

$$
\int I_{1}^{\alpha}\left(\chi_{Q} v\right)^{2}=\int_{Q} I_{2}^{\alpha}\left(\chi_{Q} v\right) v \cong \int_{Q} I_{2}\left(\chi_{Q} v\right) v
$$

for such cubes.

Proof OF THEOREM 3. (A) As in [3], it suffices to construct an $N$ dimensional subspace $S$ of $Q(H)$ such that $\int\left(|\nabla u|^{2}+\lambda|u|^{2}\right) \leq \int|u|^{2} v, u \in S$. With some computation, one verifies this inequality for

$$
S=\operatorname{Span}\left\{\theta_{\jmath} I_{2}^{\lambda}\left(\chi_{Q}, v\right)\right\}_{\jmath=1}^{N},
$$

the $\theta_{\jmath}$ being dilates and translates of a fixed $C^{\infty}$ function $\theta$ with $\theta_{\jmath} \equiv 1$ on $\frac{3}{2} Q_{\jmath}, \operatorname{supp} \theta_{\jmath} \subset 2 Q_{\jmath}, j=1, \ldots, N$.

(B) We sketch the case $\lambda=0$, following the line of proof in [3]. Thus, we prove (B) by showing that if $Q_{1}, \ldots, Q_{N}$ are all the minimal dyadic cubes satisfying $|Q|_{v}^{-1} \int_{Q} I_{2}\left(\chi_{Q} v\right) v \geq c$, then $H$ has at most $C N$ negative eigenvalues. This is done by constructing a subspace $S$ of codimension $C N$ in $L^{2}$ such that $\int|u|^{2} v \leq \int|\nabla u|^{2}, u \in S \cap Q(H)$. We define additional cubes $Q_{N+1}, \ldots, Q_{M}, M \leq C N$, and sets $E_{\jmath}, 0 \leq j \leq M$, in analogy with those in [3]. A modification of arguments in $[\mathbf{3}]$ shows that if 
$v_{j}=\chi_{E_{\jmath}} v$, then $|Q|_{v_{j}}^{-1} \int_{Q} I_{2}\left(\chi_{Q} v_{\jmath}\right) v_{j} \leq c$ for all dyadic cubes $Q$ and, thus, $\int\left(I_{1} f\right)^{2} v_{j} \leq \int f^{2}, f \in P\left(R^{n}\right), 0 \leq j \leq M$, by Theorem 1 .

It is possible to find cubes $Q_{j}^{i}$ (not necessarily dyadic or pairwise disjoint) such that $\bigcup_{i} Q_{j}^{i}=E_{\jmath}$ for $0 \leq j \leq M$ and such that the total number of $Q_{j}^{\imath}$ does not exceed $C_{n} M$. Let $S=\left\{u \in L^{2}: \int_{Q_{\jmath}^{2}} u=0\right.$ for all $\left.i, j\right\}$. Lemma 1.4 of $[\mathbf{2}]$ shows

$$
|u(x)| \leq C I_{1}\left(\chi_{Q_{\jmath}^{2}}|\nabla u|\right)(x) \leq C I_{1}\left(\chi_{E_{\jmath}}|\nabla u|\right)(x)
$$

for $x \in Q_{j}^{\imath}, u \in S$, and so

$$
\begin{aligned}
\int|u|^{2} v & =\sum_{j=0}^{M} \int|u|^{2} v_{j} \leq C \sum_{\jmath=0}^{M} \int\left[I_{1}\left(\chi_{E_{\jmath}}|\nabla u|\right)\right]^{2} v_{j} \\
& \leq \sum_{j=0}^{M} \int_{E_{\jmath}}|\nabla u|^{2}=\int|\nabla u|^{2}
\end{aligned}
$$

for $u \in S \cap Q(H)$, as required.

\section{REFERENCES}

1. B. Dahlberg, Regularity properties of Riesz potentials, Indiana Univ. Math. J. 28 (1979), $257-268$

2. E. Fabes, C. Kenig and R. Serapioni, The local regularity of solutions of degenerate elliptic equations, Comm. Partial Differential Equations 7 (1982), 77-116.

3. C. L. Fefferman, The uncertainty principle, Bull. Amer. Math. Soc. (N.S.) 9 (1983), 129-206.

4. K. Hansson, Continuity and compactness of certain convolution operators, Instiut MittagLeffler, Report No. 9, 1982.

5. H. Heinig, Weighted norm inequalities for classes of operators, preprint.

6. W. B. Jurkat and G. Sampson, On rearrangement and weight inequalities for the Fourier transform, Indiana Univ. Math. J. 32 (1984), 257-270.

7. B. Muckenhoupt, Weighted norm inequalities for the Fourier transform, Trans. Amer. Math. Soc. 276 (1983), 729-742.

8. B. Muckenhoupt and R. L. Wheeden, Weighted norm inequalities for fractional integrals, Trans. Amer. Math. Soc. 192 (1974), 251-275.

9. A. Nagel, W. Rudin and J. Shapiro, Tangential boundary behaviour of functions in Dirichlettype spaces, Ann. of Math. (2) 116 (1982), 331-360.

10. M. Reed and B. Simon, Methods of mathematical physics, Vol. I, Academic Press, New York and London, 1972.

11. E. Sawyer, A characterization of a two-weight norm inequality for maximal operators, Studia Math. 75 (1982), 1-11.

12. D. A. Stegenga, Multipliers of the Dirichlet space, Illinois J. Math. 24 (1980), 113-139.

Department of Mathematics, Brock University, St. Catharines, ONTARIO, CANADA

Department of Mathematical Sciences, McMaster University, HamilTON, ONTARIO, CANADA 